\title{
Hydroxyl radicals' production and ECG parameters during ischemia and reperfusion in rat, guinea pig and rabbit isolated heart
}

\author{
Hana Paulova ${ }^{1}$, Tibor Stracina ${ }^{2}$, Jiri Jarkovsky ${ }^{3}$, Marie Novakova $^{2}$ and Eva Taborska ${ }^{1}$ \\ ${ }^{1}$ Department of Biochemistry, Faculty of Medicine, Masaryk University, Brno, Czech Republic \\ ${ }^{2}$ Department of Physiology, Faculty of Medicine, Masaryk University, Brno, Czech Republic \\ ${ }^{3}$ Institute of Biostatistics and Analyses of Faculty of Medicine, Masaryk University, Brno, Czech Republic
}

\begin{abstract}
Ischemic and reperfusion injury is a serious condition related to numerous biochemical and electrical abnormalities of the myocardium. It has been repeatedly studied in various animal models. In this study, the production of hydroxyl radicals and electrophysiological parameters were compared in three species. Rat, guinea pig and rabbit isolated hearts were perfused according to Langendorff under strictly identical conditions. The heart rate and arrhythmia were monitored during ischemia and reperfusion periods at defined time intervals; the production of hydroxyl radical was determined by HPLC as 2.5-dihydroxybenzoic acid (2.5-DHBA) formed by salicylic acid hydroxylation. Relationship between arrhythmias and production of 2.5-DHBA was studied. The inter-species differences were observed in timing of arrhythmias onset and their severity, and in the production of 2.5-DHBA in both ischemia and reperfusion. The most considerable changes were observed in rats, where arrhythmias appeared early and with highest severity during ischemia on one side and the regular rhythm was restored early and completely during reperfusion. The corresponding changes in the production of 2.5-DHBA were observed. It can be concluded that rat isolated heart is the most suitable model for evaluation of ischemia/reperfusion injury under given experimental conditions.
\end{abstract}

Key words: Arrhythmia - Hydroxyl radical - Rat - Guinea pig - Rabbit

\section{Introduction}

Myocardial ischemia and reperfusion injury is known to be associated with biochemical, electrical and mechanical changes based on damage of cardiomyocytes (Bernier et al. 1986; Ojha et al. 2008). Enhanced generation of reactive oxygen species (ROS) and/or calcium overload have been repeatedly reported to participate in ischemia-reperfusion injury (Bolli 1990; Ferrari et al. 1991). During last two decades, the role of ROS was intensively studied. It has been demonstrated that superoxide anion radical $\left(\mathrm{O}_{2}{ }^{-}\right)$, hydrogen peroxide $\left(\mathrm{H}_{2} \mathrm{O}_{2}\right)$ and hydroxyl $\left(\mathrm{OH}^{\circ}\right)$ radicals are formed during ischemia-reperfusion injury (Zweier 1988; Bolli 1990), all coming from various sources. Xanthine oxidase, mitochondrial respiratory chain and NADPH oxidase

Correspondence to: Hana Paulova, Department of Biochemistry, Faculty of Medicine, Masaryk University, Kamenice 5, 62500 Brno, Czech Republic

E-mail: hpaulova@med.muni.cz belong to the most important systems generating radicals (Zweier et al. 2006). Generation of ROS is one of the factors which may play a role in the pathogenesis of cardiac arrhythmias often accompanying myocardial ischemia. It has been reported that ROS are involved in membrane injury leading to electrical abnormalities and arrhythmias (Bernier et al. 1986; Manning 1988; Jeong et al. 2012). ROS are produced during reperfusion period, mainly in its early phase by reintroduction of molecular oxygen into the ischemic myocardium (Bolli 1990). On the other hand, it has been observed recently that the ROS are also formed during ischemia when oxygen delivery is insufficient. Several studies have shown the formation of ROS in ischemic period in various models - in isolated guinea pig hearts (Kevin et al. 2003), in cats during brief ischemia (O'Neill et al. 1996) and in isolated cardiomyocytes during simulated ischemia (Becker 1999).

Among ROS, the hydroxyl radicals are extremely reactive and therefore are considered to be most potent ones in damaging the cells. Direct measurements of hydroxyl 
radicals have been limited primarily by the instability of these radicals. Electron paramagnetic spectroscopy (EPR) associated with spin trapping agents represents a technique usually used for the detection and determination of free radicals, including hydroxyl radicals (Zweier et al 1989). However, this method is rather expensive and requires costly instrumentation. Another approach is the use of trapping agents such as salicylic acid or phenylalanine and detection of the products of their aromatic hydroxylation (Onodera and Ashraf 1991; Sun et al. 1993).

The isolated heart perfused according to Langendorff belongs to golden standard in experimental cardiology. It enables to study various physiological as well as pathological situations including myocardial damage caused by various insults (Verdouw 1988). In the studies focused on ischemia and reperfusion, rat, guinea pig and rabbit are the most frequently employed species. However, their myocardium differs markedly, especially as the role of sarcoplasmic reticulum and equipment of cardiomyocyte membrane with specific ionic channels is concerned (Varro 1993). Moreover, the study comparing these three species at strictly identical experimental conditions during exposure to ischemia and reperfusion is missing in the literature. Therefore, in the present study we aimed to determine the most suitable animal model for evaluation of ischemia-reperfusion injury based on the electrophysiological changes and the production of high reactive hydroxyl radicals. An attention was also pointed on assessment of the relationship between arrhythmias' incidence and hydroxyl radicals' production.

\section{Materials and Methods}

\section{Chemicals}

All chemicals of analytical grade were obtained from Sigma Company (USA).

\section{Animal models}

All experiments were carried out with respect to recommendations of the European Community Guide for the Care and Use of Laboratory Animals. The experimental protocol was approved by Local Committee for Animal Treatment at Masaryk University, Faculty of Medicine.

Three species of laboratory animals, namely rat, guinea pig and rabbit, were used in this study. Fifteen Wistar rats (both sexes) weighting $277.5 \pm 47.1 \mathrm{~g}, 10$ guinea pigs (both sexes) weighting $325.7 \pm 40.2 \mathrm{~g}$, and 6 New Zealand rabbits (both sexes) weighting $3108.3 \pm 406.7 \mathrm{~g}$ were included. Rats and guinea pigs were deeply anaesthetised by inhalation of ether. After subsequent cervical dislocation, the chest was quickly opened and the heart excised with a sufficiently long segment of ascending aorta. The rabbits were pre-medicated with i.m. injection of diazepam (Apaurin, Krka) in the dose of $2 \mathrm{mg}$ and then anaesthetized by i.m. injection of mixture of xylazin (Rometar, Spofa, $2 \mathrm{mg} / \mathrm{kg}$ ) and ketamin (Narcamon, Spofa, $60 \mathrm{mg} / \mathrm{kg}$ ). Then, the animals were artificially ventilated through tracheal cannula (ventilator for small laboratory animals, World Precision Instruments, USA), the chest was opened and the heart quickly excised with sufficiently long stump of ascending aorta.

After the isolation, the heart was firmly attached to perfusion set-up via the aorta and then placed in thermostatcontrolled bath $\left(37^{\circ} \mathrm{C}\right)$ filled with Krebs-Henseleit $(\mathrm{K}-\mathrm{H})$ solution of following composition (in $\mathrm{mM}$ ): $\mathrm{NaCl} 118$, $\mathrm{NaHCO}_{3} 24, \mathrm{KCl} 4.2, \mathrm{KH}_{2} \mathrm{PO}_{4} 1.2, \mathrm{MgCl}_{2} 1.2$, glucose 5.5, Taurine 10 , and $\mathrm{CaCl}_{2}$ 1.2. The solution was continuously oxygenated with $95 \% \mathrm{O}_{2}$ and $5 \% \mathrm{CO}_{2}$. The heart was then perfused in the mode of the constant perfusion pressure (80 $\mathrm{mmHg}$ ) on Langendorff apparatus modified previously in our laboratory (Novakova et al. 2000). All hearts exhibiting any dysrrhythmias during control period were discarded from the study.

The experiment consisted of three periods, 20 minutes duration of each: first, the heart was perfused with $\mathrm{K}-\mathrm{H}$ solution containing salicylic acid ( $1 \mathrm{mmol} / \mathrm{l})$ - control period. The duration of this stabilization period was sufficient to remove anaesthetic drugs from the heart tissue. Then, the perfusion was stopped - period of ischemia (flow ischemia). Next, the flow of solution was re-introduced - reperfusion period.

\section{Electrogram recording}

During the whole experiment, touch-less electrogram was recorded from three orthogonal bipolar leads - X, Y, and $\mathrm{Z}$ (Uematsu et al. 1987). Six silver-silver chloride disc electrodes ( $4 \mathrm{~mm}$ in diameter) were placed on the inner surface of the bath in which the heart was placed during the experiment. The signals were amplified by a set of three biological amplifiers DAM50 (World Precision Instruments, USA) and further simultaneously digitized by 16-bit AD converters at a sampling rate of 2000 samples/s using a data acquisition multifunction card PCI-6250 (National Instruments, USA). Data acquisition was controlled by subroutines of a software package LabView. The digital signals were stored on a hard disc for further off-line processing.

The recorded electrograms were analysed and the heart rate (HR) changes were evaluated from manually measured and averaged ten RR intervals at the end of each $5^{\text {th }}$ minute during both ischemia and reperfusion periods. The results were then normalized to the end of control period (100\%).

The incidence of arrhythmias was noted, especially their severity, timing and frequency of appearance. Each examined heart was given a score from 0 to 5 according to scale by Haworth et al. (2010). This dysrhythmia score (DS) 
is based on Lambeth score (Walker et al. 1988) modified for a determination of the arrhythmia score per minute, by using a maximum upper ventricular tachycardia (VT) duration of $60 \mathrm{~s}$. While some VT episodes were thus split between adjacent minutes, this allowed the time course of arrhythmias to be assessed. The DS was as follows: 0, no arrhythmias; 1, $10 \mathrm{~s}$ VT or other arrhythmias, no ventricular fibrillation (VF); 2, 11-30 s VT or other arrhythmias, no VF; 3, 31-90 s VT or other arrhythmias, no VF; 4, 91-180 s VT or other arrhythmias and/or $10 \mathrm{~s}$ reversible VF; 5, $80 \mathrm{~s}$ VT or other arrhythmias and/or $10 \mathrm{~s}$ reversible VF; and 6, irreversible VF.

\section{Measurement of hydroxyl radical production}

Determination of hydroxyl radicals in perfused hearts was performed using salicylic acid trapping method (Onodera and Ashraf 1991). This method is based on the detection of the hydroxylated derivatives of the salicylic acid added to the perfused medium. It has been reported that $1 \mathrm{mmol} / \mathrm{l}$ salicylic acid can trap approximately $10 \%$ of the theoretically generated hydroxyl radicals, producing 2.3-dihydroxybenzoic acid (2.3-DHBA) and 2.5-dihydroxybenzoic acid (2.5-DHBA) and catechol in proportions of 49,40 , and $11 \%$, respectively (Floyd et al. 1984; Grootwelt and Halliwell 1986). In this study, 2.5-DHBA was used rather than 2.3-DHBA because of its greater sensitivity for quantification of hydroxyl radicals, as previously reported (Wang et al. 2004; Novakova et al. 2008). 2.5-DHBA was analysed in the coronary effluents collected at the end of the control period (after the $20^{\text {th }}$ min of stabilization) and during the $1^{\text {st }}, 5^{\text {th }}, 10^{\text {th }}, 15^{\text {th }}$, and $20^{\text {th }}$ minutes of the ischemic period and during the $1^{\text {st }}, 5^{\text {th }}, 10^{\text {th }}, 15^{\text {th }}$, and $20^{\text {th }}$ minutes of the reperfusion period. The samples were collected always during the last $10 \mathrm{~s}$ of particular interval. As a control group, rat, guinea pig and rabbit hearts - which were not subject to ischemia-reperfusion insult - were perfused for equal time $(60 \mathrm{~min})$ and electrogram recordings were done and coronary effluents were collected at the corresponding intervals. All coronary effluent samples were frozen at $-80^{\circ} \mathrm{C}$ until further analysis.

Determination of 2.5-DHBA was performed by the HPLC coupled with the electrochemical detection (McCabe et al. 1997) with our modifications (Novakova et al. 2008). The HPLC system consisted of high pressure LC-20AD pump (Shimadzu), electrochemical detector Coulochem II with analytical cell model 5010 (ESA, Chelmsford, MA, USA) and Rheodyne 7125 syringe injector (Cotati, CA, USA) with sample loop of $20 \mu \mathrm{l}$. The reversed-phase column was used (LiChrospher $100 \mathrm{RP} 18.5 \mu \mathrm{m}, 100 \times 3 \mathrm{~mm}$ I.D, Merck) with precolumn (Separon SGX, $5 \mu \mathrm{m}$ ) for the separation. Detection of 2.5-DHBA was performed by isocratic elution with the mobile phase containing $20 \%(\mathrm{v} / \mathrm{v}) \mathrm{MeOH}$ in the buffer
pH $3.6\left(0.03 \mathrm{~mol} / \mathrm{l}\right.$ citric acid and $\left.0.06 \mathrm{~mol} / \mathrm{l} \mathrm{NaH} \mathrm{NO}_{4}\right)$, flow rate $0.5 \mathrm{ml} / \mathrm{min}$. The mobile phase was filtered through nylon membrane filter $(0.2 \mu \mathrm{m}$ pore size, SolVac, USA). The analytes were detected on a dual electrode analytical cell with the first electrode set to oxidize the 2.5-DHBA at +250 $\mathrm{mV}$ and the second electrode set to oxidize salicylic acid at $+750 \mathrm{mV}$. A guard cell (model 5020) was placed between the pump and sampler at a potential of $+775 \mathrm{mV}$ to oxidize contaminants in mobile phase. The Clarity ${ }^{\mathrm{TM}}$ (Data Apex, Prague, Czech Republic) was used for the quantification of analytes.

Concentration of 2.5-DHBA in effluents during ischemic period was expressed in nmol/l. The production of 2.5DHBA during the control and the reperfusion periods was expressed as an amount of 2.5-DHBA accumulated per minute ( $\mathrm{nmol} / \mathrm{min})$.

\section{Statistical analysis}

All data are expressed as mean \pm SEM. Paired Student's ttest was applied to test significance of data in experimental group. Statistical differences of $p<0.05$ were considered to be significant. For description of continuous parameters, the average completed by $95 \%$ confidence interval was used. The relationship between the radicals' production and DS was described by Pearson correlation coefficient and its statistical significance. Statistical significance of change during ischemic and reperfusion period compared to baseline values was tested by paired $t$-test.

\section{Results}

All hearts from control group did exhibit neither electrophysiological changes (such as heart rate changes and/or arrhythmias occurrence) nor production of hydroxyl radicals.

Absolute values of $\mathrm{HR}$ at the end of control period in the hearts included in this study were $285 \pm 35,210 \pm 19$ and $157 \pm 23$ in rat, guinea pig and rabbit hearts, respectively. All hearts exposed to ischemic insult responded immediately with profound prolongation of RR intervals (Figure 1). In guinea pigs and rabbits, the normalized HR decreased to $28 \%$ and $18 \%$ of resting values at the end of ischemic period, respectively. In rat, all hearts but one even stopped beating after $5 \mathrm{~min}$ of flow ischemia. In rat and guinea pig, this decrease of HR was highly significant $(p<0.001)$, in rabbits it was less intense, however still significant $(p<0.01)$.

During the first five minutes of reperfusion, all rat hearts reinstated beating. At the end of reperfusion period, the HR reached $90 \%, 80 \%$, and $95 \%$ of resting values in rat, guinea pig, and rabbit, respectively. This HR restoration was incomplete in all species after 20 min from perfusion onset; 


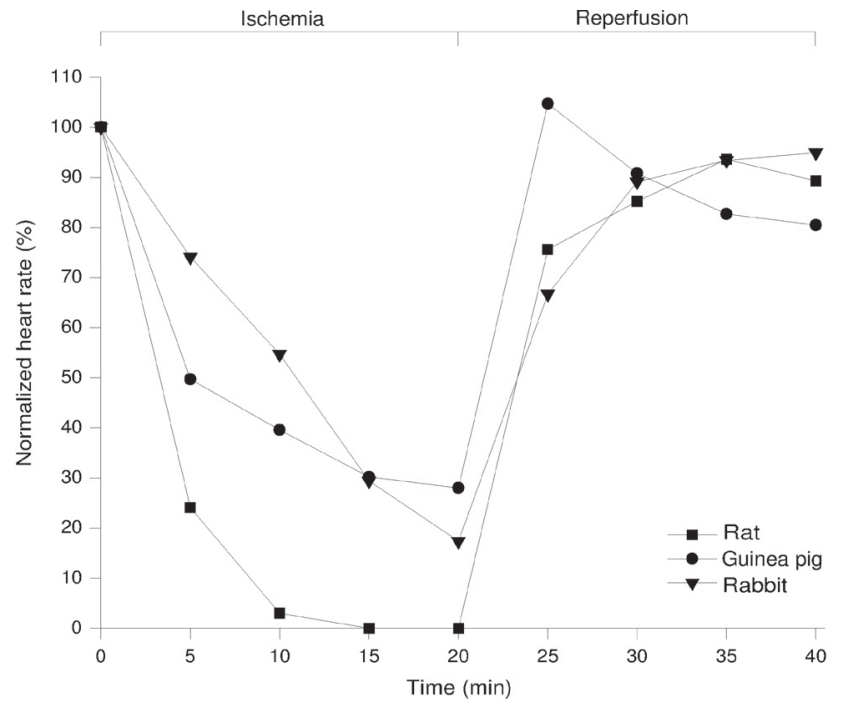

Figure 1. Heart rate changes in rat, guinea pig, and rabbit isolated hearts ( $n=12,5$ and 5 , respectively) during ischemia and reperfusion. Normalized to the end of control period.

however, the differences from the values at the end of control period were insignificant.

All examined hearts were then classified according to the most severe arrhythmia observed during each experimental period. The values of modified DS are overviewed in Figure 2. As the inter-species differences are concerned, it is obvious that rat hearts respond to ischemia early and with the highest intensity (see Figure 2). On the other hand, the arrhythmias disappear during the first few minutes of reperfusion. The guinea pig hearts respond to ischemia a couple of minutes later and with lower intensity; however, they do not recover from ischemia during the whole reperfusion period (score around 1 at the end of the reper- fusion). The rabbit hearts react even later, after ten minutes of ischemia, and their full recovery takes approximately 10 min of reperfusion.

As noted above, in rat hearts the incidence of arrhythmias during ischemia was high and the arrhythmias were severe. The most frequent was ventricular fibrillation; it was present in all studied hearts and the total duration of this type of arrhythmia was $47 \%$ of ischemic period. Also premature ventricular beats and episodes of AV block of the second and the third degree were frequently observed. All hearts restored spontaneous beating during reperfusion. Most arrhythmias in reperfusion phase were detected within first 10 minutes - ventricular fibrillation ( $6 \%$ of ischemia duration), frequent premature ventricular beats (24 per one heart on average) and short episodes of ventricular tachycardia.

In guinea pig hearts, the most frequent arrhythmia was AV blockade of the second and the third degree, present exclusively during ischemia ( $25 \%$ of ischemia period). Shortlasting episodes of ventricular fibrillation were present in two animals out of five; they terminated spontaneously. During reperfusion, very frequently single or multiple premature ventricular beats were observed (47 per one heart on average). In one case, short ventricular tachycardia appeared.

The most frequent arrhythmia in rabbit hearts was AV blockade of the second and third degree, present almost entirely in the second part of ischemia and lasting $20 \%$ of ischemia duration. In reperfusion period, premature ventricular beats (three animals) and short ventricular tachycardia (one case) were observed.

The basal levels of 2.5-DHBA collected during the $20^{\text {th }}$ minute of control period were negligible in all isolated hearts. The values of 2.5-DHBA concentration produced during 20 minutes of ischemia are shown in Figure 3. It is obvious that remarkable increase of 2.5-DHBA concentration was detected already after the first minute of ischemia in
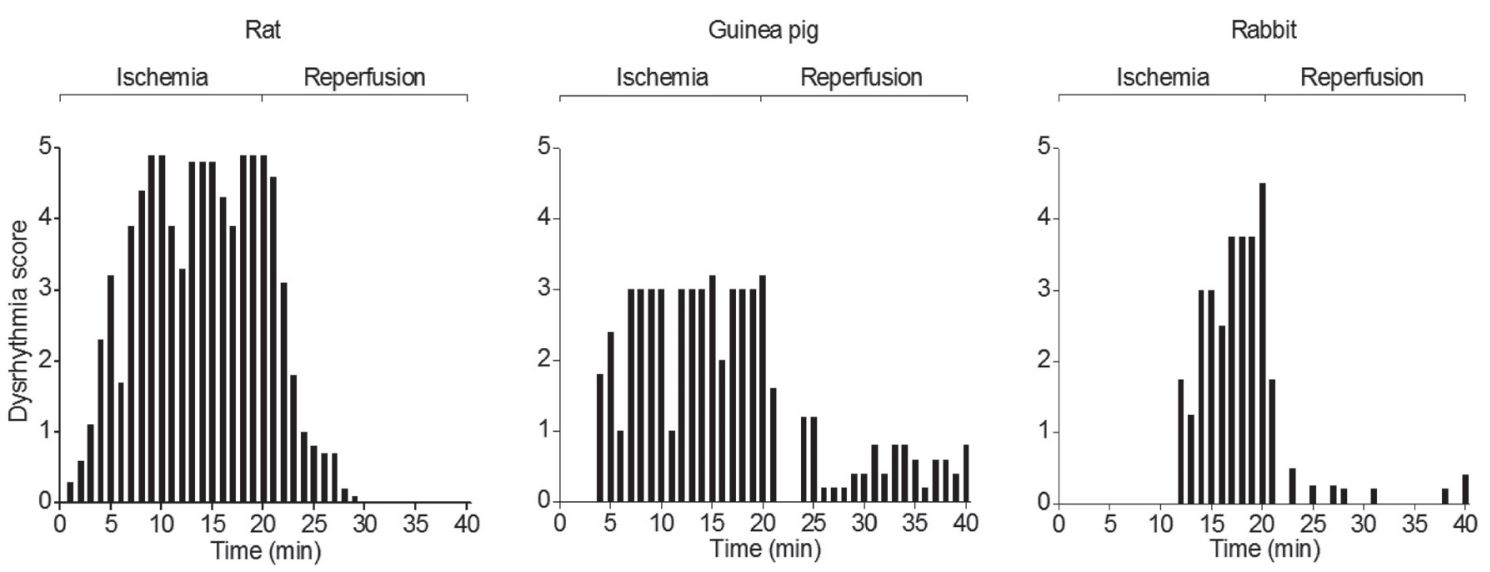

Figure 2. Dysrhythmia score of rat, guinea pig, and rabbit isolated hearts ( $n=12,5$, and 5 , respectively) during particular minutes of ischemia and reperfusion. 

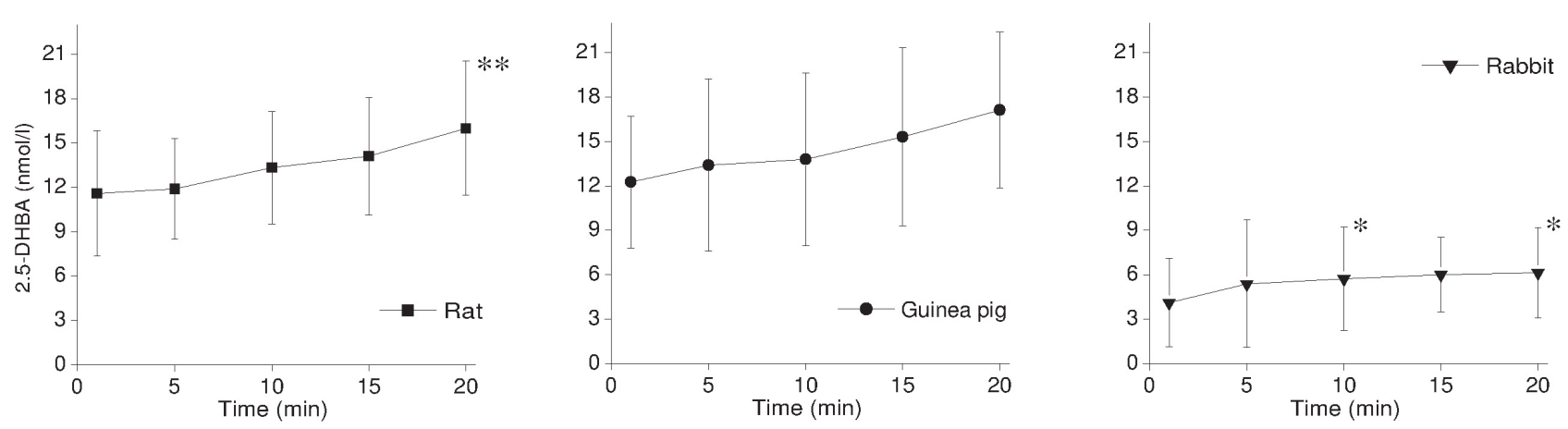

Figure 3. Production of 2.5-DHBA in rat, guinea pig and rabbit $(n=15,10$, and 6 , respectively) during ischemic period. The results are presented as a mean, 95\% CI. Differences of $p<0.05$ were considered statistically significant. ${ }^{*} p<0.05 v s$. the first minute of ischemia, ${ }^{* *} p<0.01 v s$. the first minute of ischemia.

all examined hearts $(11.587 \pm 8.376 \mathrm{nmol} / \mathrm{l}, 12.252 \pm 7.200$ $\mathrm{nmol} / \mathrm{l}$, and $4.117 \pm 2.850 \mathrm{nmol} / \mathrm{l}$ in rats, guinea pigs and rabbits, respectively). The final concentrations with values of $15.991 \pm 8.977 \mathrm{nmol} / \mathrm{l}, 17.119 \pm 8.510 \mathrm{nmol} / \mathrm{l}$, and 6.135 $\pm 2.919 \mathrm{nmol} / \mathrm{l}$ were obtained during the $20^{\text {th }}$ minute of ischemia in rat, guinea pig, and rabbit group, respectively. Thus statistically significant, although low increase of 2.5DHBA between the $1^{\text {st }}$ and the $20^{\text {th }}$ minute was found only in rats and rabbits.

During the reperfusion period the values are expressed as an amount of 2.5-DHBA accumulated per minute (Figure 4) because the experiments were performed at the constant perfusion pressure and the flow of the perfusate through coronary system was not constant. The highest amounts of 2.5-DHBA were detected during the $1^{\text {st }} \mathrm{min}$ of reperfusion in all examined hearts $(0.038 \pm 0.020 \mathrm{nmol} / \mathrm{min}, 0.224 \pm$ $0.141 \mathrm{nmol} / \mathrm{min}$, and $0.059 \pm 0.034 \mathrm{nmol} / \mathrm{min}$ in rats, guinea pigs and rabbits, respectively). Then, the concentration of 2.5-DHBA was decreasing during reperfusion in all three groups and the lowest values of 2.5-DHBA were obtained at the end of the reperfusion period. The amounts of 2.5DHBA during the $20^{\text {th }}$ minute of reperfusion were $0.016 \pm$ $0.011 \mathrm{nmol} / \mathrm{min}, 0.206 \pm 0.157 \mathrm{nmol} / \mathrm{min}$, and $0.053 \pm 0.029$ $\mathrm{nmol} / \mathrm{min}$ in rats, guinea pigs and rabbits, respectively. Only in rat, considerable decrease $(p<0.001)$ of the production of hydroxyl radicals was observed. To compare the production of hydroxyl radicals during ischemia and at the beginning of reperfusion, 2.5-DHBA in the $1^{\text {st }}$ minute of reperfusion was expressed also in nmol/l $(5.70 \pm 3.42 \mathrm{nmol} / \mathrm{l}, 28.26 \pm$ $14.38 \mathrm{nmol} / \mathrm{l}, 3.88 \pm 2.22 \mathrm{nmol} / \mathrm{l}$ in rat, guinea pig and rabbit groups, respectively).

In order to evaluate relationship between the 2.5-DHBA concentration and arrhythmias incidence, the averaged DS (calculated for particular time interval) and subsequent production of 2.5-DHBA has been expressed as Pearson coefficient for both ischemia and reperfusion periods. In ischemia, relationship between averaged DS and corresponding 2.5-DHBA concentration was found in all species.
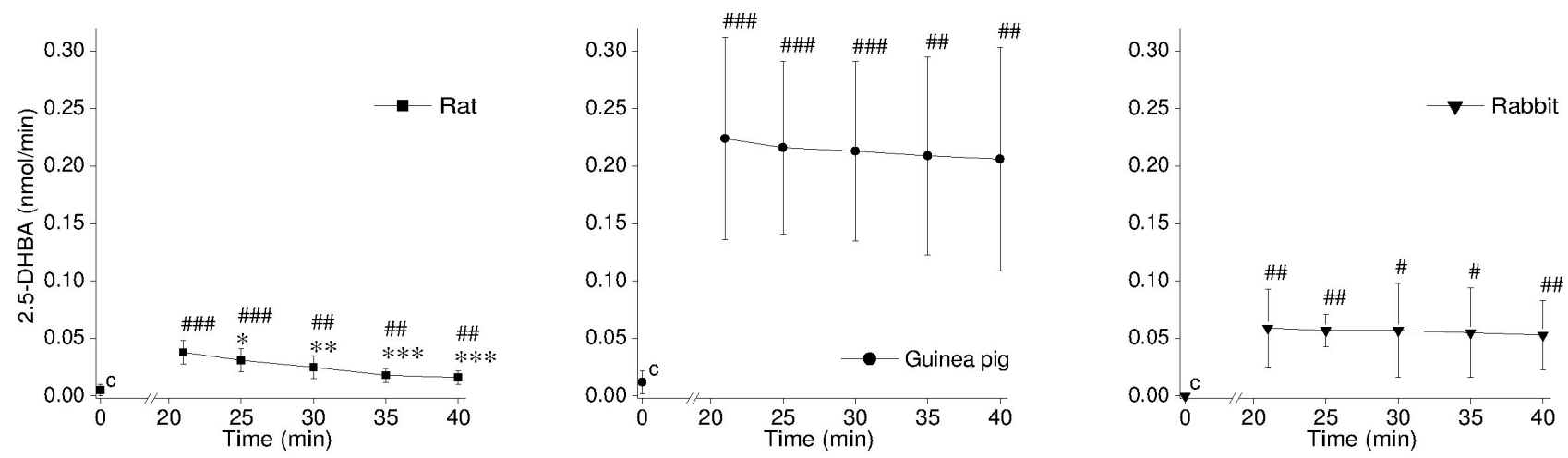

Figure 4. Accumulated amount of 2.5-DHBA in rat, guinea pig and rabbit isolated hearts ( $n=15,10$, and 6 , respectively) during 20 min of reperfusion period. The results are presented as a mean, 95\% CI. Differences of $p<0.05$ were considered statistically significant. c, control value; ${ }^{*} p<0.05 v$ s. the first minute of reperfusion; ${ }^{* *} p<0.01 v s$. the first minute of reperfusion; ${ }^{* * *} p<0.001 v s$. the first minute of reperfusion; ${ }^{\#} p<0.05$ vs. control period; ${ }^{\# \#} p<0.01$ vs. control period; ${ }^{\# \#} p<0.001$ vs. control period. 
In rat hearts, DS $(6-10 \mathrm{~min})$ is related to 2.5 -DHBA (in the $\left.10^{\text {th }} \mathrm{min}\right), \mathrm{r}=0.422$. In guinea pig hearts, DS (11-15 $\mathrm{min}$ ) is related to $2.5-\mathrm{DHBA}$ (in the $15^{\text {th }} \mathrm{min}$ ), $\mathrm{r}=0.879$. In rabbit hearts, DS (11-15 $\mathrm{min})$ is significantly related to 2.5-DHBA (in the $15^{\text {th }} \mathrm{min}$ ), $\mathrm{r}=0.436(p<0.001)$ and DS $\left(16-20 \mathrm{~min}\right.$ ) is related to $2.5-\mathrm{DHBA}$ (in the $20^{\text {th }} \mathrm{min}$ ), $\mathrm{r}=$ 0.799 . In reperfusion period, the relationship between DS and 2.5-DHBA concentration was found in guinea pigs. In these hearts, DS (11-15 min) is related to 2.5 -DHBA (in the $\left.15^{\text {th }} \min \right), r=0.354$.

\section{Discussion}

In this study, the production of hydroxyl radical and electrogram parameters were evaluated during ischemiareperfusion injury in the model of isolated heart perfused according to Langendorff. Special attention has been paid to inter-species differences. It is worth mentioning that all experiments at isolated heart - electrogram recording and perfusate collection - were performed at strictly identical conditions.

The changes of HR and the incidence, severity and timing of arrhythmias were analysed from electrogram recorded by touch-less method. Significant slowing of spontaneous cardiac activity has been observed in all hearts. This result was expected and served for verification of our model, especially as a proof of sufficient duration of ischemic period. No inter-species differences have been found in this part of the study.

As the arrhythmias are concerned, marked differences were present namely in timing of arrhythmias onset and their severity. These inter-species differences can be most probably attributed to different equipment of cardiac cells with specific ionic channels. Their impaired gating during ischemia-reperfusion may result in increased arrhythmias incidence. Rat, guinea pig and rabbit hearts differ mainly in repolarization part of action potential. Guinea pig ventricular cardiomyocytes do not develop transient outward current; on the other hand, it is present in rat and in about half of the rabbit cardiac ventricular cells. Delayed rectifier current of quite high amplitude is reported in guinea pig cardiomyocytes, but it is negligible or even absent in rat and rabbit. Inward rectifier potassium current is similar in rabbit and guinea pig (Varro et al. 1993). Thus, different response to ischemia and reperfusion in our experiments may be caused by the effect on characteristics of delayed rectifier and/or transient outward currents.

Increased production of ROS in ischemic animal heart has been reported in various models (O'Neill et al. 1996; Becker 1999; Kevin et al. 2003) and it has been explained as a consequence of derangement of the mitochondrial electron transport system and decrease of energy status due to lack of oxygen (Dhalla and Duhamel 2007). Among ROS, hydroxyl radicals are the most reactive ones. Salicylic trapping method was chosen for their detection in our experiment (Onodera and Ashraf 1991) and production of 2.5-DHBA was determined as a measure of hydroxyl radical formation.

During the ischemia onset remarkable increase of 2.5DHBA concentration was detected in all examined hearts, indicating presence of oxidative stress. During the following 19 min, further increase was detected in all hearts; however, it was statistically significant only for rats and rabbits. These values are comparable with results of Bao et al. (2007) who reported that in rabbits the production of 2.5-DHBA measured by microdialysis technique reached during $30 \mathrm{~min}$ of ischemia was in tens of nmol/l.

During the reperfusion period, the production of 2.5DHBA was measured in the first minute and then in each consecutive 5-min interval. The highest values of 2.5-DHBA concentration in effluent were obtained in the first minute; however, these values at least partially corresponded to 2.5DHBA that was accumulated in the perfusate during the previous ischemic period. Nevertheless, the increased level of 2.5-DHBA in the effluent after the $5^{\text {th }}$ minute of reperfusion and later can be ascribed to its new formation and implicates the production of hydroxyl radicals also as a consequence of reperfusion. This finding corresponds with previous reports where reintroduction of oxygen into an ischemic heart is related to accelerated production of ROS in the first few minutes of reperfusion (Zweier et al. 1988; Cai et al. 1995; Ravingerova et al. 1999; Merril G. 2001). It is difficult to assess whether the production of hydroxyl radicals was higher during ischemia or at the beginning of reperfusion since the experiment was done at constant perfusion pressure and thus the coronary flow was not controlled. Therefore, the values of 2.5-DHBA were expressed in different units in ischemia and reperfusion periods. In order to compare at least partially 2.5-DHBA production, we expressed its values - measured in the first minute of reperfusion - also in nmol/l. Taken from this point of view, only in guinea pig higher production of hydroxyl radicals was detected in reperfusion than in ischemia. The production of 2.5-DHBA during on-going reperfusion had descending character in all three groups and the lowest values of 2.5-DHBA were obtained at the end of the reperfusion period. Only in rat, considerable decrease of the production of hydroxyl radicals during 20 min reperfusion was observed. This inter-species difference might indicate that rat hearts are more resistant to reperfusion injury and their recovery is therefore faster.

Also other inter-species differences have been observed in our model. In ischemia, the production of hydroxyl radicals was at least twice higher in rat and guinea pigs then in rabbits. During reperfusion, the hydroxyl radical production was lower in rabbit and rat comparing to guinea pig. In the 
first minute of reperfusion, production of hydroxyl radicals was around 5 times higher in guinea pig than in the other two studied species and the increased values persisted over all reperfusion period. These differences might be explained by various sensitivity of animal hearts to the oxidation stress and ischemia-reperfusion injury.

It was found in this study that the incidence of ischemic and reperfusion arrhythmias is associated with the production of hydroxyl radicals. In ischemia, tight relationship between dysrhythmia score and 2.5-DHBA was observed in the hearts of all studied species. The same relationship was observed in guinea pig hearts during reperfusion. These results indicate that the hydroxyl radicals might participate in the pathogenesis of arrhythmias. It corresponds with study of Ravingerova et al. (1999) who described that reperfusion induced arrhythmias are coincided with generation of $\mathrm{H}_{2} \mathrm{O}_{2}$.

In this paper, the model for studying ischemia-reperfusion injury in isolated heart perfused according to Langendorff was proposed and used under strictly identical experimental conditions in three species of laboratory animals. Since the response to ischemia-reperfusion insult monitored by arrhythmias incidence, their severity and timing, hydroxyl radicals' production and recovery from ischemia was most considerable in rat isolated heart, it may be concluded that rat heart is the most suitable model for evaluation of ischemiareperfusion injury in this experimental model. Moreover, tight relationship between the arrhythmias incidence and severity and hydroxyl radicals' production in this experimental model has been proven.

Acknowledgement. This work was supported by the grant project MSM0021622402 of the Ministry of Education of the Czech republic and SV MUNI MUNI/A/0818/2012 and MUNI/ A/0951/2012.

\section{References}

Bao N., Minatoguchi S., Kobayashi H., Yasuda S., Kawamura I., Iwasa M., Yamaki T., Sumi S., Misao Y., Arai M., Nishigaki K., Takemura G., Fujiwara T., Fujiwara H. (2007): Pravastatin reduces myocardial size via increasing protein kinase C-dependent nitric oxide, decreasing oxyradicals and opening the mitochondrial adenosine triphosphate-sensitive potassium channels in rabbits. Circ. J. 71, 1622-1628 http://dx.doi.org/10.1253/circj.71.1622

Becker L. B., Vanden Hoek T. L., Shao Z. H., Li C. Q., Schumacker P. T. (1999): Generation of superoxide in cardiomyocytes during ischemia before reperfusion. Am. J. Physiol. Heart Circ. Physiol. 277, H2240-2246

Bernier M., Hearse D. J., Manning A. S. (1986): Reperfusion-induced arrythmias and oxygen-derived free radicals. Circ. Res. 58, 331-340 http://dx.doi.org/10.1161/01.RES.58.3.331
Bolli R. (1990): Mechanism of myocardial "stunning". Circulation 82, 723-738 http://dx.doi.org/10.1161/01.CIR.82.3.723

Cai Q., Takemura G., Ashraf M. (1995): Antioxidative properties of histidine and its effect on myocardial injury during ischemia/ reperfusion in isolated rat heart. J. Cardiovasc. Pharmacol. $25,147-155$ http://dx.doi.org/10.1097/00005344-199501000-00023

Dhalla N. S., Duhamel T. A. (2007): The paradoxes of reperfusion in the ischemic heart. Heart Metab. 37, 31-34

Ferrari R., Ceconi C., Curello S., Cargnoni A., Pasini E., De Giulli F., Albertini A. (1991): Role of oxygen free radicals in ischemic and reperfused myocardium. Am. J. Clin. Nutr. 53, S215-222

Floyd R. A., Watson J. J, Wong P. K. (1984): Sensitive assay of hydroxyl free radical formation utilizing high pressure liquid chromatography with electrochemical detection of phenol and salicylate hydroxylation products. J. Biochem. Biophys. Methods 10, 221-235 http://dx.doi.org/10.1016/0165-022X(84)90042-3

Grootwelt M., Halliwell B. (1986): Aromatic hydroxylation as a hydroxyl-radical formation in vivo. Biochem. J. 237, 499-504

Haworth R. A., Potter K. T., Russell D. C. (2010): Role of arachidonic acid, lipoxygenase, and mitochondrial depolarization in reperfusion arrhythmias. Am. J. Physiol., Heart Circ. Physiol. 299, H165-174

http://dx.doi.org/10.1152/ajpheart.00906.2009

Jeong E. M, Liu J., Liu M., Study M., Gao G., Varghese S. T., Sovari A. A., Dudlei Jr. S. C. (2012): Metabolic stress, reactive oxygen species, and arrhythmia. J. Mol. Cell Cardiol. 52, 454-463 http://dx.doi.org/10.1016/j.yjmcc.2011.09.018

Kevin L. G., Camara A. K. S., Riess M. L., Novalija E., Stowe D. F. (2003): Ischemic preconditioning alters real-time measure of $\mathrm{O} 2$ radicals in intact hearts with ischemia and reperfusion. Am. J. Physiol., Heart Circ. Physiol. 284, H566-574

Manning A. S. (1988): Reperfusion - induced arrhythmias: do free radicals play a critical role? Free Radic. Biol. Med. 4, 305-316 http://dx.doi.org/10.1016/0891-5849(88)90051-2

Merrill G., McConnell P., Vandyke K., Powell S. (2001): Coronary and myocardial effets of acetaminophen: protection during ischemia-reperfusion. Am. J. Physiol., Heart Circ. Physiol. 280, $\mathrm{H} 2631-2638$

McCabe D. R, Maher J. T, Acworth I. N. (1997): Improved method for the estimation of hydroxyl free radical levels in vivo based on liquid chromatography with electrochemical detection. J. Chromatogr. B 691, 23-32

http://dx.doi.org/10.1016/S0378-4347(96)00416-1

Novakova M., Moudr J., Braveny P. (2000): A modified perfusion system for pharmacological studies in isolated hearts. In: Analysis of Biomedical Signals and Images. pp 162-164, Vutium Press, Brno

Novakova M., Bardonova J., Provaznik I., Taborska E., Bochorakova H., Paulova H., Horky D. (2008): Effect of voltage sensitive dye di-4-ANEPPS on guinea pig and rabbit myocardium. Gen. Physiol. Biophys. 27, 45-54

Ojha N., Roy S., Radtke J., Simonetti O., Gnyawali S., Zweier J. L., Kuppusamy P., Sen Ch. K. (2008): Characterization of the structural and functional changes in the myocardium following 
focal ischemia-reperfusion injury. Am. J. Physiol., Heart Circ. Physiol. 294, H2435-2443 http://dx.doi.org/10.1152/ajpheart.01190.2007

O’Neill Ch. A., Fu L. W., Halliwell B., Longhurst J. C. (1996): Hydroxyl radical production during myocardial ischemia and reperfusion in cats. Am. J. Physiol., Heart Circ. Physiol. 271, H660-667

Onodera T., Ashraf M. (1991): Detection of hydroxyl radicals in the post-ischemic reperfused heart using salicylate as a trapping agent. J. Mol. Cell. Cardiol. 23, 365-370 http://dx.doi.org/10.1016/0022-2828(91)90072-T

Ravingerova T., Slezak J., Tribunova N., Dzurba A., Uhrik B., Ziegelhoffer A. (1999): Free oxygen radicals contribute to high incidence of reperfusion-induced arrythmias in isolated rat hearts. Life Sci. 65, 1927-1930 http://dx.doi.org/10.1016/S0024-3205(99)00449-X

Sun J. Z., Kaur H., Halliwell B., Li X. Y., Bolli R. (1993): Use of aromatic hydroxylation of phenylalanine to measure production of hydroxyl radicals after myocardial ischemia in vivo. Circ. Res. 73, 534-549 http://dx.doi.org/10.1161/01.RES.73.3.534

Uematsu T., Vozeh S., Ha H. R., Follath F., Nakashima M. (1987): Method for stable measurement of the electrocardiogram in isolated guinea pig heart. J. Pharmacol. Methods 18, 179-185 http://dx.doi.org/10.1016/0160-5402(87)90010-6

Varro A., Lathrop D. A., Hester S. B., Nanasi P. P., Papp J. G. Y. (1993): Ionic currents and action potentials in rabbit, rat, and guinea pig ventricular myocytes. Basic Res. Cardiol. 88, 93-102
Verdouw P. D., van den Doel M. A., de Zeeuw S., Duncker D. J. (1998): Animals models in the study of myocardial ischemia and ischaemic syndromes. Cardiovasc. Res. 39, 121- 135 http://dx.doi.org/10.1016/S0008-6363(98)00069-8

Walker M. J. A., Curtis M. J., Hearse D. J., Campbell R. W. F., Janse M. J., et al. (1988): The Lambeth Conventions: guidelines for the study of arrhythmias in ischaemia, infarction, and reperfusion. Cardiovasc. Res 22, 447-455 http://dx.doi.org/10.1093/cvr/22.7.447

Wang N., Minatoguchi S., Chen X. H., Arai M., Uno Y., Lu C., Takemura G., Fujiwara T., Fujiwara H. (2004): Antidiabetic drug miglitol inhibits myocardial apoptosis involving decreased hydroxyl radical production and Bax expression in an ischemia/reperfusion rabbit heart. Br. J. Pharmacol. 142, 983-990 http://dx.doi.org/10.1038/sj.bjp.0705863

Zweier J. L. (1988): Measurements of superoxide-derived free radicals in the reperfused heart. J. Biol. Chem. 25, 1353-1357

Zweier J. L., Kuppusamy P., Williams R., Rayburn B. K., Smith D., Weisfeldt M. L., Flauberty J. T. (1989): Measurement and characterisation of postischemic free radical generation in the isolated perfused heart. J. Biol. Chem. 32, 18890-18895

Zweier J. L., Hassan Talukder M. A. (2006): The role of oxidants and free radicals in reperfusion injury. Cardiovasc. Res. 70, 181-190

http://dx.doi.org/10.1016/j.cardiores.2006.02.025

Received: April 21, 2012

Final version accepted: November 20, 2012 